\title{
Speed Control of Fuzzy PID Intelligent Cars with Self-Tuning Factors
}

\author{
Wenjuan Zhang ${ }^{1}$, Haomiao $\mathrm{Ma}^{2}$ and Rui Gao ${ }^{1}$ \\ ${ }^{1}$ Department of Electrical Engineering, Baoji University of Arts and Sciences, \\ Shaanxi, China \\ ${ }^{2}$ School of Computer Science, Shaanxi Normal University, Shaanxi, China \\ Zhangwj19811130@163.com
}

\begin{abstract}
Speed control of intelligent cars requires fast response speeds and good dynamic performance. According to the different requirements of the speed controller in the starting and steady state, a fuzzy PID controller with self-tuning factors was proposed in this paper. In the designed controller, fuzzy controller with a two-input and three-output structure was adopted to track the set speed, two self-tuning factors $\alpha_{1}$ and $\alpha_{2}$ were select can distribute different tuning factors according to the system error in order to realize self-tuning of the fuzzy control rule. Simulation results using MATLAB/SIMULINK showed that the proposed fuzzy controller had a faster response speed, no overshoot, and a strong resistive capacity to external disturbances. Therefore, this controller can drive intelligent cars to move along a set track at an optimum speed.
\end{abstract}

Keywords: Intelligent car, speed control, fuzzy control, adaptive tuning factor

\section{Introduction}

The intelligent car is also called a wheeled robot. It has an integrated intelligent system [1] with the functions of an environmental monitor, automatic following of a guide line, and self-tuning according to the environment. In each kind of industry and even in daily life, there is a wide application prospect for the intelligent car.

Research into movement speed control of intelligent cars has been a recent hotspot of technological development in the field of mobile robot control theory. At present, the most widely used control technology relies mainly on traditional PID control [2]. Although the traditional PID algorithm allows for simple control, the strength of this control depends on how accurately the control object can be mathematically modeled and on the initial PID setting parameters [3]. An accurate mathematical model is very difficult to establish due to the complex working environment of intelligent cars with various interferences and influences in their paths. If traditional PID control is not improved, it will be very difficult to obtain adequate control of intelligent cars $[4,5]$. Therefore, intelligent control has been proposed for speed control of intelligent cars, and the most representative theory is that of fuzzy control. Fuzzy PID control is a type of nonlinear control that imitates human control, to a certain degree. It does not require an accurate model of the control object. According to input/output data of actual systems, and referring to the operating experiences of site operation personnel, it is able to control the system in real-time [6, 7]. The scholar E.H. Mamdani achieved great success in this field. He designed a fuzzy PID controller by adopting the rule language of fuzzy control and he applied this to an industrial intelligent car. In the end, fuzzy control played a critical role [8]. The State Key Laboratory of Intelligent Technology in Qinghua University successfully applied fuzzy PID control theory to speed control of an intelligent car, which showed good adaptive capacity to the

Received (August 6, 2016), Review Result (January 13, 2017), Accepted (February 6, 2017) 
environment during actual operation [9]. Based on the above-referenced studies, the purpose of this study was to analyze the fuzzy control unit of an intelligent car with a DC motor speed control system. A speed control algorithm of a fuzzy PID intelligent car with an adaptive self-tuning factor was also proposed. This algorithm introduced a self-tuning factor, $\alpha$, into the fuzzy PID controller, which allowed the weights of various inputs to be tuned in different phases, such as start up, stop, and steady-state running. This greatly improved the accuracy, stability, and reaction speed of the intelligent car's speed control. The effectiveness of the algorithm was verified using the simulation base of MATLAN/SIMULINK.

\section{System Structure and Speed Control Strategy of an Intelligent Car}

\subsection{System Structure of an Intelligent Car [10-12]}

The system structure of an intelligent car is shown in Figure 1. It primarily consists of six parts: a car-mounted controller, a wireless communication device, a geomagnetic detection sensor, a motor drive unit, a power supply and detection unit, and a car alarm. Among them, the motor drive unit is driven by a DC motor. It is primarily responsible for control of the car speed and carrying out independent following of the track. The basic principle of an independent following track is that the detecting system of the path is utilized to identify and follow a black guide line on a track so that autonomous driving can be achieved. Performance of the car in this task is proportional to the speed of the car. Once it detects that the black guide line is in any side of the car body, the sensor will decide that the current path is a curve track. In that moment, the control system is required to tune the speeds of the right and left wheels in the shortest time, so that the car body can be turned. Otherwise, the car will rush out off the track. Therefore, the performance of the car mainly depends on the control accuracy and response time of the car speed.

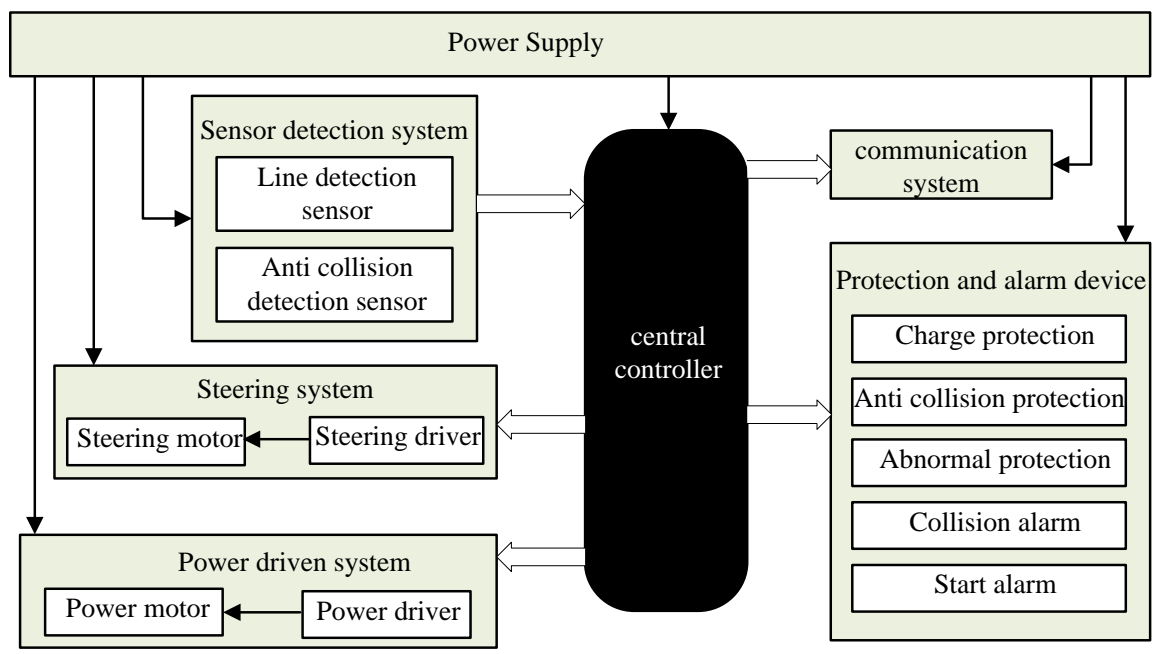

Figure 1. The System Structure of the Intelligent Car

\subsection{Speed Control Strategy of an Intelligent Car}

Based on the above analysis of the car body structure, and in order to ensure that the intelligent car can accurately follow the path, the speed of the car needs to be constantly tuned in real-time under different running environments. Traditional PID control has a high accuracy and good steady-state performance. However, it is not capable of adapting to different road conditions (i.e. strict straight, straight, small curve, large curve, and sharp curve). Fuzzy control systems have good dynamic performances, but their steady-state accuracies are poor as oscillations of polar rings may occur. Therefore, we proposed a 
combination of these two types of control. By combining the fuzzy PID control with the structural characteristics of an intelligent car system, the speed control strategy of an intelligent car was obtained, as shown in Figure $2[13,14]$.

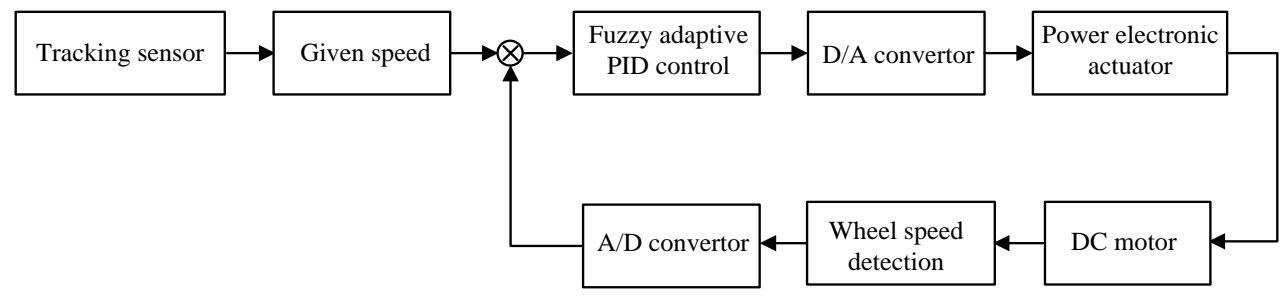

Figure 2. The Speed Control Strategy of the Intelligent Car

The system adopted a fuzzy PID adaptive closed-loop algorithm to control the speed of a DC motor. The driving controller controlled the work of an infrared sensor and obtained the reference speed of the car from the collected road-tracking information. Meanwhile, the speed detection module of the wheel detected the speed of the car and compared this with the reference speed after isolation, amplification, and A/D conversion. If the error in speed was decreased or increased significantly, this indicated that the path was a curve with an upward or downward slope. This signal of speed error was sent to the fuzzy PID controller. The controller implemented the selection of a fuzzy rule, outputted accurate speed pulse signals, and sent signals into the actuator of the power electronics. This allowed the DC motor to run accurately, according to the tracking speed from actual road conditions.

\section{Fuzzy PID Design of the Speed Control in Intelligent Car}

The so-called fuzzy adaptive PID controller constantly detected $e$ and $e c$ on the basis of traditional PID control according to the fuzzy relationship between the three parameters of $\mathrm{P}$, I \& $\mathrm{D}$, the error, $e$, and the error variation rate of $e c=d e / d t$. Through the pre-determined fuzzy relationship, and by using the fuzzy reasoning method, the three parameters of the PID controller were modified in real-time to make the PID parameters autonomously set according to the signals of the sensor, and to make the output parameter values have smaller amplitudes of variation during the period. The fuzzy adaptive PID controller effectively resolved the interference problem of output mutation or error variation, which led to stronger adaptability [15-16]. The typical structure of the controller was divided into two main parts: fuzzy reasoning and traditional PID control. The structure diagram is shown in Figure 3.

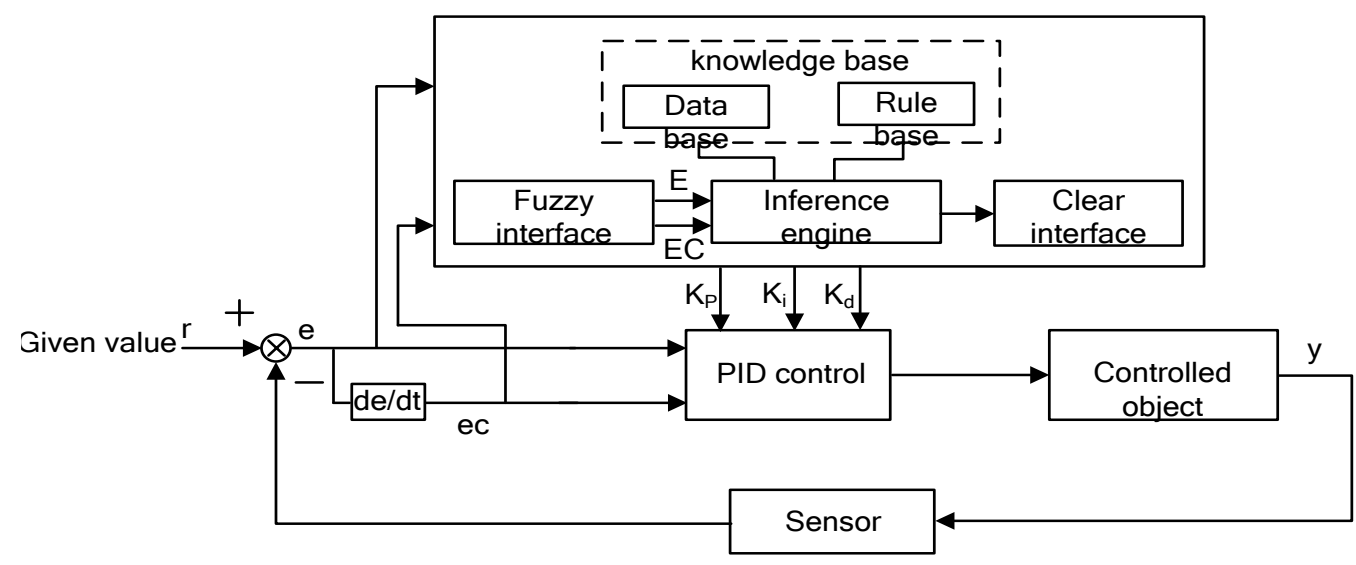

Figure 3. The Structure Diagram of Fuzzy Adaptive Tuning PID Controller 
According to the control structure shown in Figure 3, the fuzzy PID design of the speed control in an intelligent car was divided into the following four steps [17-18]:

First Step: Determine the input/output variables of the system.

The intelligent car adopted a fuzzy controller with a two-input and three-output structure to track the set speed. The input variables were set as the DC motor's error of angular speed, $e$, and the variation rate of error, $e c$, while the output variables were $\Delta \mathrm{K}_{\mathrm{p}}$, $\Delta \mathrm{K}_{\mathrm{i}}$ and $\Delta \mathrm{K}_{\mathrm{d}}$. According to field test data of DC motors on site, and considering the error of speed sensor, the input parameters $(e$ and $e c$ ) were set to -5 and 5 , respectively. According to the system setting results of the DC motor, the basic discourse domain of $\Delta \mathrm{K}_{\mathrm{p}}, \Delta \mathrm{K}_{\mathrm{i}}$ and $\Delta \mathrm{K}_{\mathrm{d}}$ was determined to be $[-2,2]$.

Second Step: The input and output variables fuzzification.

Only through fuzzification, the input of the fuzzy controller can be used to control the output solution. The larger the quantization level of a variable, the higher the accuracy of the fuzzy control and the larger the required amount of calculations. For these reasons, the quantization levels of $e, e c, \Delta \mathrm{K}_{\mathrm{p}}, \Delta \mathrm{K}_{\mathrm{i}}$ and $\Delta \mathrm{K}_{\mathrm{d}}$ were all set to the $13^{\text {th }}$ level in this design; the discourse domain of two input and one output in the fuzzy set were: $\{-6,-5,-4,-3,-2$, $-1,0,+1,+2,+3,+4,+5,+6\}$. In this set, the elements expressed large negative, medium negative, small negative, zero, small positive, medium positive, and large positive values, respectively ${ }^{[5]}$. All the corresponding fuzzy languages were $\{\mathrm{NB}, \mathrm{NM}, \mathrm{NS}, \mathrm{ZO}, \mathrm{PS}, \mathrm{PM}$, $\mathrm{PB}\}$, where NB means a sharp speed down, NM means a medium speed down, NS means a small speed down, ZO means maintaining the current speed, PS means a small speed up, PM means a medium speed up, and PB means a sharp speed up.

After the conversion of each variable from the basic domain into the fuzzy domain, the next step was to define the fuzzy subset which corresponded to each fuzzy language variable. The fuzzy subset was defined by setting the shape of the membership function curve of the fuzzy subset. The determined membership function curve was discretizated and the subset of corresponding fuzzy variables was structured. During the speed control of intelligent cars, triangle membership functions were all adopted for the fuzzy subsets of input/output variables because the generation of speed error was random and to simplify the calculations. The values of the fuzzy variables were set according to recorded driving experiences, and the membership functions [6] of e, ec, $\Delta \mathrm{K}_{\mathrm{p}}, \Delta \mathrm{K}_{\mathrm{i}}$ and $\Delta \mathrm{K}_{\mathrm{d}}$, which belonged to each fuzzy subset in the discourse domain, were obtained as shown in Figure 4.

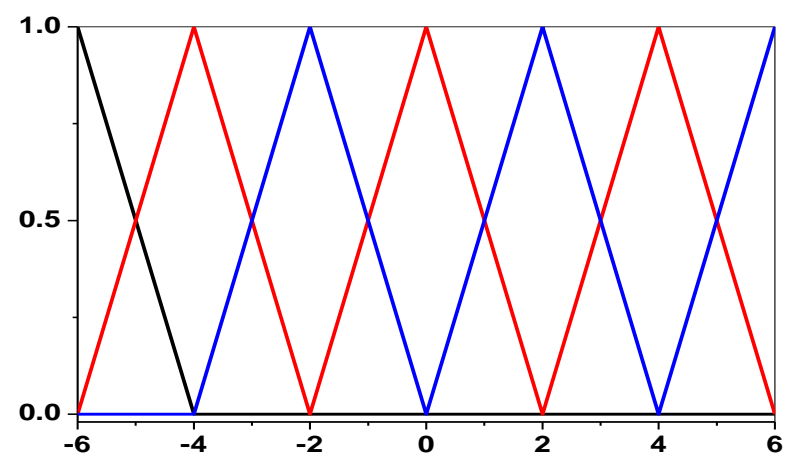

Figure 4. The Diagram of Membership Function

Third Step: Determine the fuzzy control rule of PID parameters.

The fuzzy control rule was used to determine the relationships between the three PID parameters and $e$ as well as $e c$ at different time points. It continuously detected $e$ and $e c$ during running, and it modified the three parameters of $\Delta \mathrm{K}_{\mathrm{p}}, \Delta \mathrm{K}_{\mathrm{i}}$ and $\Delta \mathrm{K}_{\mathrm{d}}$ on-line according to the fuzzy control theory, so as to satisfy the different requirements of different $e$ and $e c$ on the control parameters. Thus, the controlled object can have good dynamic and static performance. The control rule of speed error, $e$, is especially important 
for the application of intelligent cars. Control without this rule will result in oscillation of the calculated speed, inability to appropriately adapt to different road conditions, and a reduction in the running stability of the car. When the error, $e$, between the actual moving speed of the car body and the expected speed is bigger, a larger $\mathrm{K}_{\mathrm{p}}$ should be used in order to increase the tracking speed of the system. However, a smaller $K_{d}$ should be used in the beginning when there is a sudden increase in deviation resulting in differential over saturation. This allowed the control function to stay within its permitted range. Furthermore, the integral action should be limited in order to prevent a large overshoot in the speed response of the system and generation of integral saturation. In general, $\mathrm{K}_{\mathrm{i}}$ is set to equal 0 . According to the operational experience of intelligent cars, the fuzzy control rule of output parameters $\left(\Delta \mathrm{K}_{\mathrm{p}}, \Delta \mathrm{K}_{\mathrm{i}}\right.$ and $\left.\Delta \mathrm{K}_{\mathrm{d}}\right)$ may be obtained in the speed control of intelligent cars, which is shown in Table 1. There are a total of 49 pieces of these rules. The final output values of $\Delta \mathrm{K}_{\mathrm{p}}, \Delta \mathrm{K}_{\mathrm{i}}$ and $\Delta \mathrm{K}_{\mathrm{d}}$ may be obtained by using the rule table via the fuzzy solution. Under different circumstances, the system will call different control rules to adjust the speed of the motor, therefore realizing the goal of the speed control in the car.

Table 1. The Fuzzy Control Rule of $\Delta k_{p}, \Delta k_{i}, \Delta k_{d}$

\begin{tabular}{|c|c|c|c|c|c|c|c|}
\hline $\begin{array}{l}\Delta \mathrm{K}_{\mathrm{p}} \\
\Delta \mathrm{K}_{\mathrm{i}} \\
\Delta \mathrm{K}_{\mathrm{d}}\end{array}$ & & & & $\mathrm{Ec}$ & & & \\
\hline $\mathrm{e}$ & NB & NM & $\mathrm{NS}$ & $\mathrm{ZO}$ & PS & $\mathrm{PM}$ & PB \\
\hline NB & $P B \backslash N B \backslash P S$ & $P B \backslash N B \backslash N S$ & $P M \backslash N M \backslash N B$ & $P M \backslash N M \backslash N B$ & PS\NS\NB & ZOIZO\NM & ZO\ZO\PS \\
\hline NM & $P B \backslash N B \backslash P S$ & $P B \backslash N B \backslash N S$ & $P M \backslash N M \backslash N B$ & $P S \backslash N S \backslash N M$ & PS\NS\NM & ZOLZOLNS & NSIZOIZO \\
\hline NS & $P M \backslash N B \backslash Z O$ & $P M \backslash N M \backslash N S$ & $P M \backslash N S \backslash N M$ & $P S \backslash N S \backslash N M$ & ZOLZOLNS & NS\PS\NS & NS\PS\ZO \\
\hline $\mathrm{ZO}$ & PM\NM\ZO & $P M \backslash N M \backslash N S$ & PS\NS\NS & ZOIZOINS & NS\PS\NS & $\mathrm{NM} \backslash \mathrm{PM} \backslash \mathrm{NS}$ & $\mathrm{NM} \backslash \mathrm{PM} \backslash \mathrm{ZO}$ \\
\hline PS & PS\ZO\PB & PSINSIZO & ZO\ZO\ZO & NS\PS\ZO & NS\PS\ZO & NM\PM\ZO & $N M \backslash P B \backslash Z O$ \\
\hline PM & PS\ZO\PB & ZOLZOINS & NS\PS\PS & NM\PS\PS & $\mathrm{NM} \backslash \mathrm{PM} \backslash \mathrm{PS}$ & $\mathrm{NM} \backslash \mathrm{PB} \backslash \mathrm{PS}$ & $\mathrm{NB} \backslash \mathrm{PB} \backslash \mathrm{PB}$ \\
\hline $\mathrm{PB}$ & ZO\ZO\PB & ZO\ZO\PM & $\mathrm{NM} \backslash P S \backslash P M$ & $\mathrm{NM} \backslash \mathrm{PM} \backslash \mathrm{PM}$ & $\mathrm{NM} \backslash \mathrm{PM} \backslash \mathrm{PS}$ & $\mathrm{NB} \backslash P B \backslash P S$ & $\mathrm{NB} \backslash \mathrm{PB} \backslash \mathrm{PB}$ \\
\hline
\end{tabular}

Fourth Step: Clearness of fuzzy variables

The output variables of $\mathrm{K}_{\mathrm{p}}, \mathrm{K}_{\mathrm{i}}$ and $\mathrm{K}_{\mathrm{d}}$ obtained from fuzzy reasoning are the fuzzy variables, and cannot be directly used to control the speed of the DC motor. They must first be transformed into accurate variables using a reasonable method. As the gravity method could reflect the real distribution of control variables, this was adopted to transform the fuzzy variables into accurate variables in this design.

\section{The Design of a Fuzzy PID Controller with Self Tuning Factors}

The following features were be concluded by analyzing the fuzzy PID design steps described above. If the quantization levels of error, $e$, error variety rate, $e c$, and the output control variables, $\Delta \mathrm{K}_{\mathrm{p}}, \Delta \mathrm{K}_{\mathrm{i}}$ and $\Delta \mathrm{K}_{\mathrm{d}}$ (which are recorded as $\mathrm{U}$ uniformly), were divided into one same level, for example:

$$
\{E\}=\{E C\}=\{U\}=\{-m,-m+1, \ldots,-2,-1,0,+1,+2, \ldots, m-1, m\}
$$

Then, their control query table could be approximated by the following relationship expressed as $[19,20]$ :

$$
\mathrm{U}=-(\mathrm{E}+\mathrm{EC}) / 2
$$

Two observations were made from the above formula. First, in the conventional two-dimensional fuzzy controller, the output variable values depended on the input variables of $e$ (error) and $e c$ (error variation), and weight coefficients for both of these parameters were 0.5 . Second, the relationship of the control rule in the two-dimensional fuzzy controller described in this formula was fixed and could not be tuned. Therefore, once the design was finished, the control rule of the design was also fixed.

Aiming at this limitation, we considered introducing a tuning factor, $\alpha$, into Formula (1), such that:

$$
\mathrm{U}=-[\alpha \mathrm{E}+(1-\alpha) \mathrm{EC}] \quad \alpha \in(0,1)
$$


It may be observed in Formula 2 that through tuning the value of $\alpha$, the function levels of error, E, and error variation rate, EC, on the output variable $\mathrm{U}$ will be changed to some extent. Through this method, the control rule could be turned.

For the fuzzy PID controller with a two-input and one-output structure in the speed control of an intelligent car, the speed error, E, is often bigger during the initial speed control phase. At this time, it is expected that the action of the error value would be larger than that of the error variation rate in the control rule. In contrast, when the speed control process is in steady state, the system error is smaller. The main task of control system is to reduce the overshoot, and to make the system stable as soon as possible, which required the action of the error variation rate to be larger in the control rule. However, once the tuning factor, $\alpha$, is selected, it cannot be changed; therefore, it is difficult to adapt and satisfy self-tuning of the car's speed using the fuzzy control rule. Thus, two tuning factors, $\alpha_{1}$ and $\alpha_{2}$, were selected in this study. When the speed error, E, is smaller, and $|E| \leq \frac{m}{2}$ is set, the control rule was tuned by $\alpha_{1}$. When the speed error, E, is larger, and $|E|>\frac{m}{2}$ is set, the control rule was tuned by $\alpha_{2}$. The specific adjustment is shown in Formula (3). The structure of the fuzzy PID controller with self tuning factors is shown in Figure 5. Combined with traditional fuzzy PID control, the software flow chart with self tuning factors is shown in Figure 6. In the Figure, the input variables as before, were set as the DC motor's error of angular speed $e$, and the variation rate of error $e c$.

$$
U=\left\{\begin{array}{l}
-\left[\alpha_{1} E+\left(1-\alpha_{1}\right) E C\right],|E| \leq \frac{m}{2} \\
-\left[\alpha_{2} E+\left(1-\alpha_{2}\right) E C\right],|E|>\frac{m}{2}
\end{array} \alpha_{1}, \alpha_{2} \in(0,1), \alpha_{1}<\alpha_{2}\right.
$$

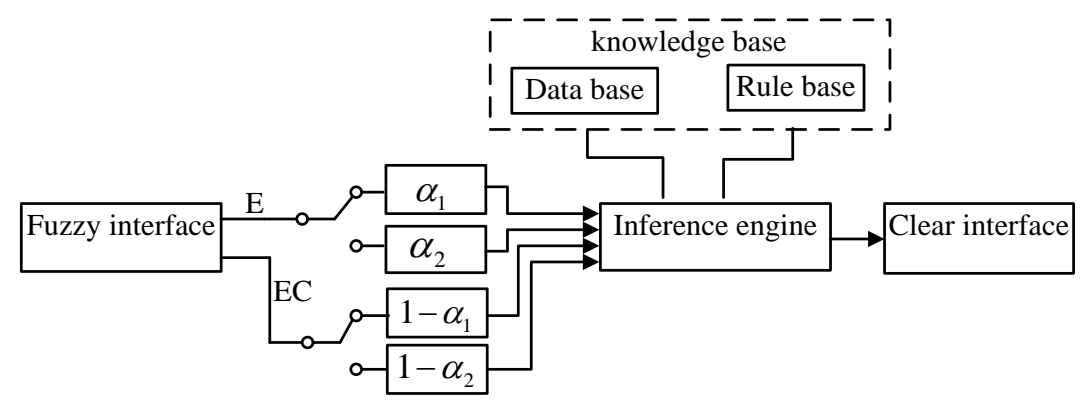

Figure 5. The Structure of Fuzzy PID Controller with Self Tuning Factors 


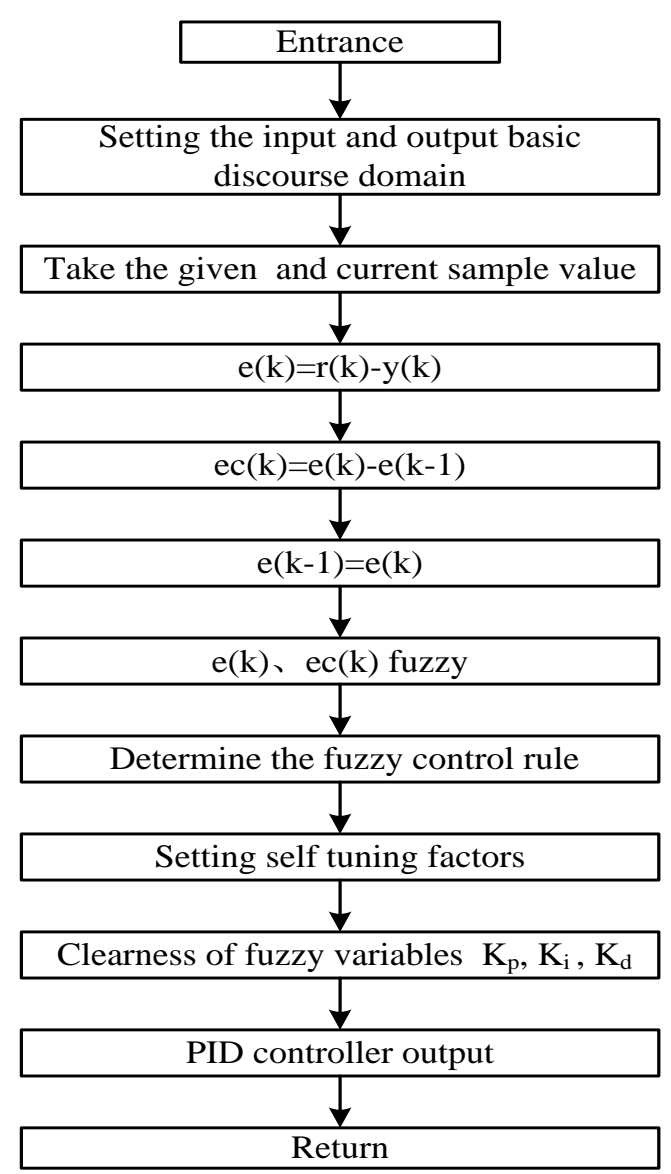

Figure 6. The Software Flow Chart of Fuzzy PID Control with Self Tuning Factors

\section{Simulation Verification}

In order to verify the effectiveness of the fuzzy PID algorithm with self-tuning factors in the speed control of an intelligent car proposed in this study, the simulation verification was carried through on the platform of MATLAB.

First, the mathematical model of the DC motor was established. Based on the electronic and dynamic formulas of the car's drive motor, the mathematical formula describing the DC motor was simplified as:

$$
G(S)=\frac{400}{s^{2}+50 s}
$$

In MATLAB, the fuzzy PID design results of the speed control in the intelligent car were as follows:

(1) FIS Editor. According to the analysis in section four, the intelligent car adopted the fuzzy controller of a two-input and three-output structure to track the set speed. The input variables were the speed error, $e$, and the error variation rate, $e c$, of the DC motor, while the output variables were $\Delta \mathrm{K}_{\mathrm{p}}, \Delta \mathrm{K}_{\mathrm{i}}$ and $\Delta \mathrm{K}_{\mathrm{d}}$. The design results are shown in Figure 7 . 


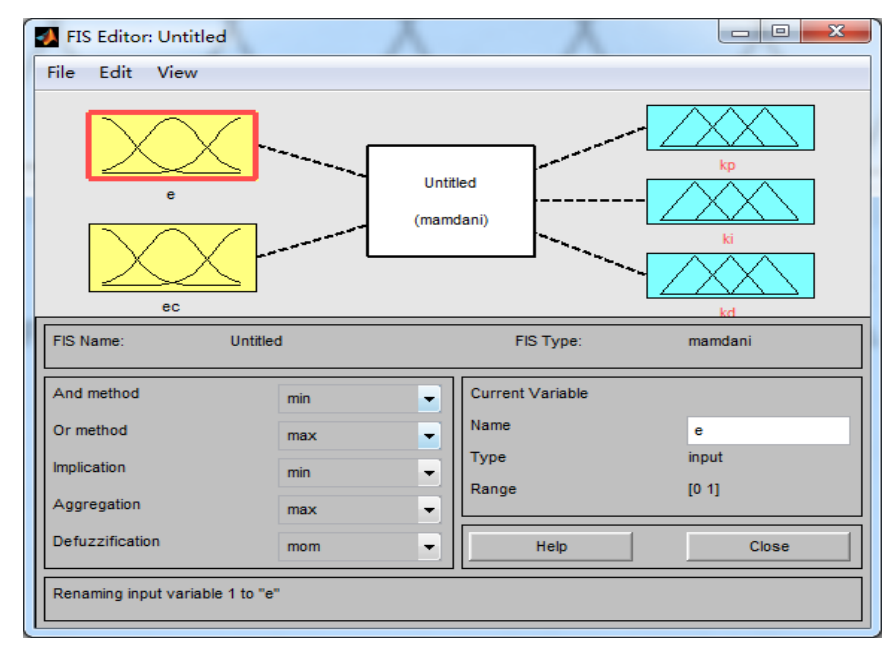

Figure 7. FIS Editor Design

(2) Establishment of membership function. Triangle functions were selected for the membership functions of input and output variables established in the FIS editor, which is shown in Figure 8.

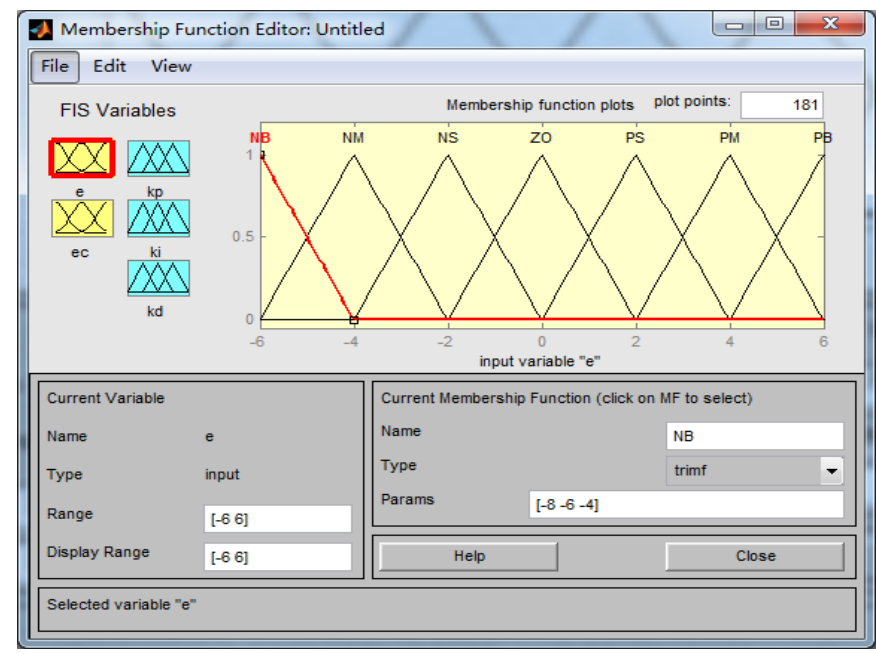

Figure 8. Membership Function Design

(3) Establishment of Fuzzy Rule Base. The 49 pieces of fuzzy control rule bases established for the output parameters of the speed control for the intelligent car are shown in Figure 9. 


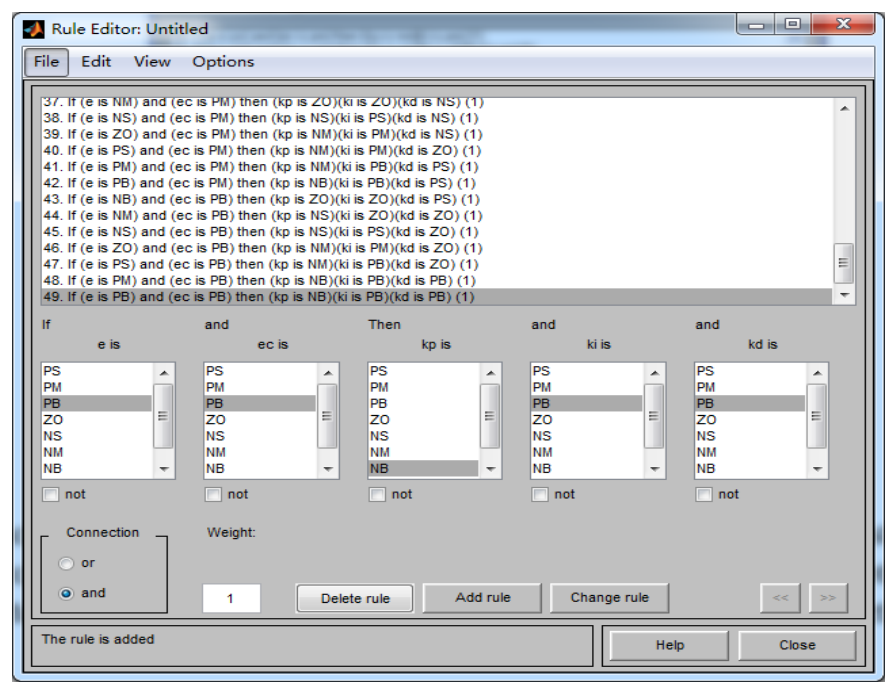

Figure 9. Control Rules Design

(4) Rule Viewer. The obtained rule viewer is shown in Figure 10.

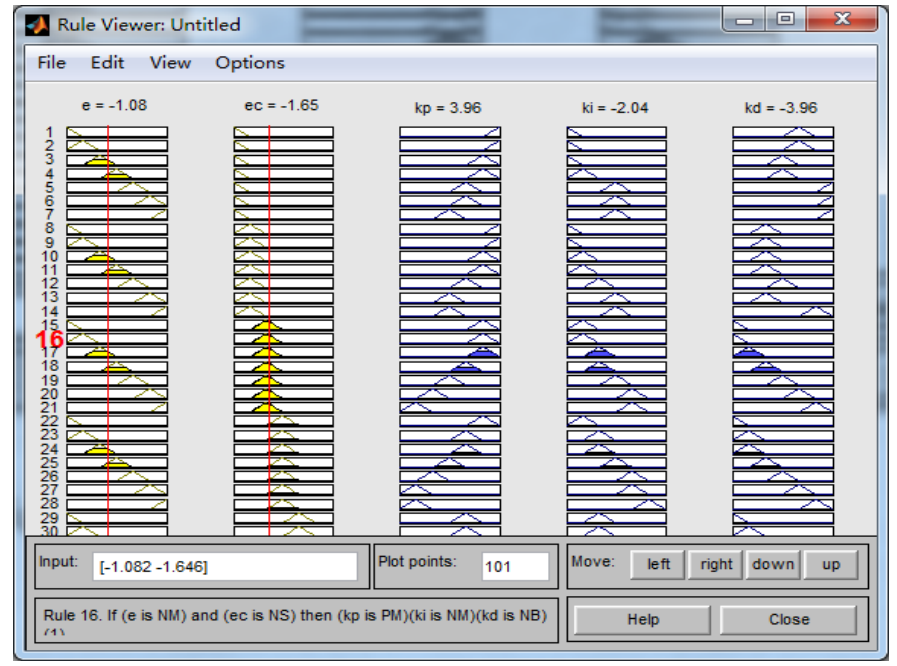

Figure 10. Rule Viewer

(5) Simulation Model of MATLAB/Simulink. The fuzzy PID simulation model with self-tuning factors is shown in Figure 11. In this model, the fuzzy factor of $\left|\mathrm{K}_{\mathrm{e}}\right|$ was $0.005,\left|\mathrm{~K}_{\mathrm{ec}}\right|$ was 0.01 and the s-function was the function written by the self-tuning factor algorithm. Optimization of these factors can improve the control effect of the fuzzy controller. After repeated verifications, this design determined that the control effect of the car's speed was optimized when $\alpha_{1}$ equaled 0.7 and $\alpha_{2}$ equaled 0.3 . Thus, in the speed control system, the tuning factors were 0.7 and 0.3 .

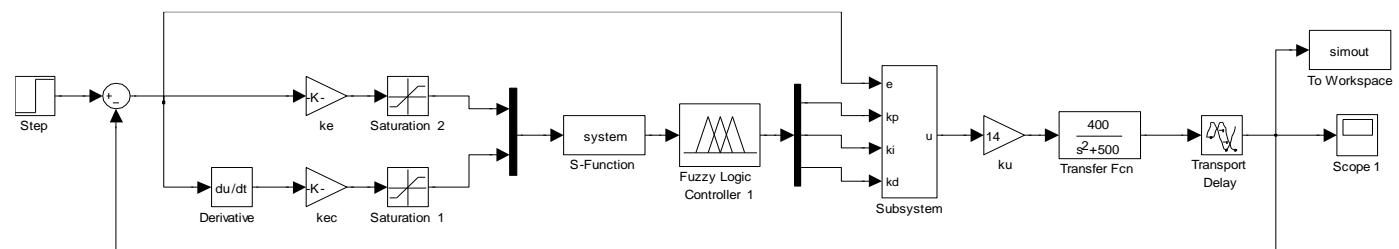

Figure 11. The System Simulation Block Diagram 
(6) Simulation Results \& Analysis. In order to explain the advantages and disadvantages of this algorithm, the simulations were implemented for traditional PID control, conventional fuzzy PID control, and fuzzy PID control with self-adjusted factors, while the control performances of all three controls were compared. Inside, the red, green, and blue curves represent the simulation results of traditional PID, conventional fuzzy PID, and fuzzy PID with self-adjusted factors, respectively.

The response curves of the three kinds of control algorithms are shown in Figure 12. It may be observed that the adjusting time for conventional PID is approximately $6 \mathrm{~s}$ with a maximum overshoot of $42 \%$ and steady state tends to be achieved after only 3 semi-oscillating periods. The response time for conventional fuzzy PID control is reduced to approximately $2.4 \mathrm{~s}$ with an overshoot of $23 \%$ and steady state tends to be achieved after 1 period of oscillation. For fuzzy PID control with self-tuning factors, different tuning factors can be distributed according to the error value. Therefore, its response time is approximately $1 \mathrm{~s}$, and steady state was achieved without any oscillation or overshoot. Figure 13 is an amplified drawing of the simulation results after the three control algorithms were all in the steady states and the disturbance of 10 was added in when $t=$ $10 \mathrm{~s}$. It may be observed that the following error is present for both conventional PID and conventional fuzzy PID control under the outside interference. The adjustment error of the fuzzy PID with self-tuning factors was small after the controller was disturbed and the transient state process was achieved without any oscillation.

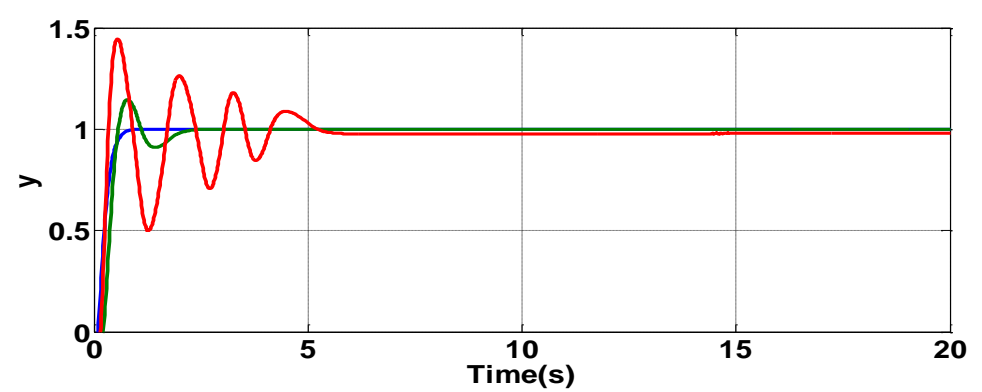

Figure 12. The Response Curves of the Three Kinds of Control Algorithms

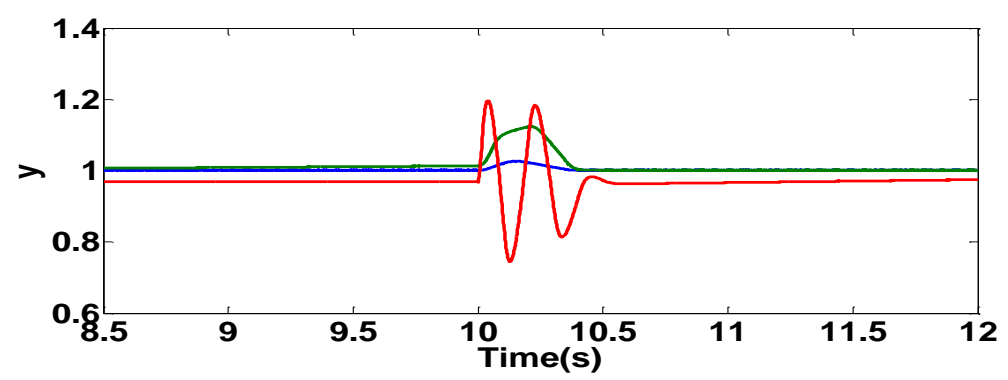

Figure 13. The Amplified Drawing of the Simulation Results after Adding the Disturbance

\section{Conclusions}

With the goal application of the speed control of intelligent cars, this paper proposed a fuzzy PID speed controller with self-tuning factors, and reached the following conclusions:

(1) Compared to the traditional PID and the conventional fuzzy PID, the response time of the fuzzy PID speed controller with self-tuning factors was reduced. During its response process, there was no oscillation or overshoot. This controller had better dynamic and steady-state performances.

(2) After two self-adjusting factors were introduced into the speed controller of the 
conventional fuzzy PID, the fuzzy controller could distribute different tuning factors according to the value of system error. Thus, the control rule could be changed, and the resisting disturbance capacity of the output response was significantly strengthened. During actual motion of a car, it could travel along a racing track at the optimum speed more stably. This controller has very practical applications for real-world use of intelligent cars.

\section{Acknowledgments}

This work was supported by National Nature Science Foundation of China (51207002), the Industrial Science and Technology Research of Shaanxi Province(2013K07-12), the Natural Science Basic Research Plan in Shaanxi Province of China (2014JM8347), the Technology transfer to promote engineering project of $\mathrm{Xi}$ 'an bureau of science and technology (CXY1347(4)).

\section{References}

[1] H. Li, X. Jing and H. Lam, "Fuzzy Sampled-Data Control for Uncertain Vehicle Suspension Systems", Cybernetics IEEE Transactions on, vol. 44, no. 7, (2014), pp. 1111-1126.

[2] M. L. Zhou, D. K. Lu and W. M. Li, "Optimized Fuzzy Logic Control Strategy for Parallel Hybrid Electric Vehicle Based on Genetic Algorithm”, Mechanical Engineering, Materials Science and Civil Engineering, vol. 274, (2013), pp. 345-349.

[3] J. Mendes, R. Araujo and T. Matias, "Automatic extraction of the fuzzy control system by a hierarchical genetic algorithm", Engineering Applications of Artificial Intelligence, vol. 29, no. 3, (2014), pp. 70-78.

[4] Y. S. H. Najjar and M. M. B. Amer, "Using a smart device and neuro-fuzzy control system as a sustainable initiative with green cars", Journal of the Energy Institute, vol. 89, no. 2, (2016), pp. 256-263.

[5] Y. B. Cui, J. Zheng and Y. T. Ju, "Precise Angle Control of Electromechanical Actuator with Fuzzy PID and Genetic Algorithm”, Mechatronics \& Applied Mechanics II, vol. 32, no. 2, (2013), pp. 88-98.

[6] Y. .J. Xin, "Control Algorithm Research on Smart Car Based on Fuzzy PID”, Experiment Science and Technology, vol. 14, no. 1, (2016), pp. 46-48.

[7] D. Y. De, C. G. Chao and Y. X. Long, "Controller Design for Automated Guided Vehicle Based on Posture Analysis", Chinese Journal of Automotive Engineering, vol. 6, no. 3, (2016), pp. 168-174.

[8] E. H. Mamdani and P. J. King, "The application of fuzzy control systems to industrial processes", Automatica, vol. 13, no. 3, (1997), pp. 235-242.

[9] H. Lia, X. Wang and S. Song, "Vehicle Control Strategies Analysis Based on PID and Fuzzy Logic Control”, Procedia Engineering, vol. 137, (2016), pp. 234-243.

[10] H. Guanfu, L. Yugong and F. Jingjing, "Optimization Design of the Dual-Steering Control System For All-Wheel Independent Electric Drive Vehicle", Electric Information and Control Engineering (ICEICE), International Conference on 2011, (2011).

[11] H. M. Li, X. B. Wang and S. B. Song, "Vehicle Control Strategies Analysis Based on PID and Fuzzy Logic Control”, Procedia Engineering, vol. 137, (2016), pp. 234-243.

[12] B. Xiong and Q. U. Shiru, "Intelligent Vehicle's Path Tracking Based on Fuzzy Control", Journal of Transportation Systems Engineering and Information Technology, vol. 10, no. 2, (2010), pp. 70-75.

[13] W. I. Gabr, "A new approach for automatic control modeling, analysis and design in fully fuzzy environment", Ain Shams Engineering Journal, vol. 6, no. 3, (2015), pp. 835-850.

[14] H. Khayyam, "Adaptive intelligent control of vehicle air conditioning system", Applied Thermal Engineering, vol. 51, no. 1-2, (2013), pp. 1154-1161.

[15] S. Kanarachos and A. Kanarachos, "Intelligent road adaptive suspension system design using an experts' based hybrid genetic algorithm”, Expert Systems with Applications, vol. 42, no. 21, (2015), pp. $8232-8242$.

[16] S. Cai, M. Becherif and M. Wack, "Wireless control of automotive actuator based on PID and Fuzzy Logic", IFAC Proceedings Volumes, vol. 44, no. 1, (2015), pp. 9745-9750.

[17] X. Wang, M. Fu and H. Ma, "Lateral control of autonomous vehicles based on fuzzy logic", Control Engineering Practice, (2015), pp. 1-17.

[18] M. A. C. Fernandes, "Fuzzy controller applied to electric vehicles with continuously variable transmission", Control Engineering Practice, (2015), pp. 1-17.

[19] K. B. Chvatil, "Genetic algorithm-based optimal fuzzy control system for the MT 25 microtron", Journal of Instrumentation, vol. 8, no. 5, (2013), pp. 1058-1069.

[20] H. Wang and B. C. C. Lin, "Adaptive fuzzy control for pure-feedback stochastic nonlinear systems with unknown dead-zone input", International Journal of Systems Science, vol. 45, no. 12, (2013), pp. 2552-2564. 


\section{Authors}

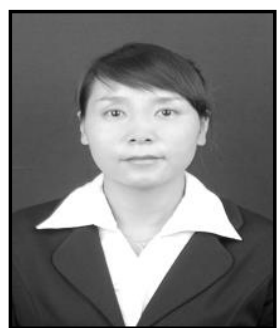

Wenjuan Zhang, was born in Shaanxi, China.She received her B.S. degree in Automation Engineering, and her M.S. and Ph.D. degrees in Power Electronics and Electrical Drives from the Xi'an University of Technology,Shaanxi, China, in 2004, 2007 and 2011, respectively. From 2007 to 2011, she was with Wind Power Generation Technology, Xi'an, China. In 2012, she joined the Baoji University of Arts and Sciences, Baoji, China, as an Associate Professor. Her current research interests include wind power generation, AC-AC converters, and PWM converter/inverter systems. 